\title{
Prácticas contemporáneas para potenciar la gestión del talento humano en el contexto Hondureño, año 2016-2017
}

Jesús Argueta Moreno ${ }^{1}$

\section{RESUMEN}

Esta investigación desarrolla un análisis cualitativo sobre el fenómeno denominado "networking profesional", el cual presenta distintas particularidades en el contexto hondureño y más aún en el ámbito de Tegucigalpa. Destacando que el "networking profesional" junto con otras prácticas y condiciones, es una herramienta clave para potenciar, acelerar y asegurar el éxito laboral, aun en condiciones profesionales muy extremas. La cual tiende a mal interpretarse y asociarse con la jerga popular en Honduras, a nivel del concepto de "compadrazgos". No obstante este documento pretende eliminar esta errada concepción y facilitar aquellas prácticas sistémicas que le permiten a profesional, contar con un potencial de trabajo superior al de la media.

Por otro lado, luego de evaluar de manera cualitativa algunas características del "networking profesional" en el contexto local, es importante aseverar, que conforme a la evidencia empírica, la cual esta acuerpada por insumos teóricos, se infiere que la principal limitante para la efectiva gestión del "networking profesional" en el mercado laboral de Tegucigalpa, responde a la escasa percepción de fortaleza institucional, de las organizaciones públicas y privadas en el país, situación que compromete la aplicación de las buenas prácticas del "netwoking profesional", así como la sana búsqueda de oportunidades de trabajo.

Finalmente, se destaca que la pesquisa más allá de definir las distintas particularidades del "networking" profesional" en Honduras, demuestra que el principio del Antropólogo Robin Dunbar no es aplicable con la realidad laboral en el país.

Palabras clave: empleabilidad, networking, mercado laboral.

${ }^{1}$ Profesor de la Facultad de Ciencias Económicas Administrativas y Contables (FCEAC), Universidad Nacional Autónoma de Honduras (UNAH): jesus.argueta@unah.edu.hn 


\section{ABSTRACT}

The present investigation had to develop a qualitative analysis on the phenomenon called "Professional Networking", presents different peculiarities along the Honduran context. The document highlights' the "Professional Networking" practices and conditions, that promote carrer boosting and job assurance, even in very extreme conditions. The term tends to be misinterpreted and associated with the popular jargon of Hondurans, at the level of the concept of "compadrazgos". However, this document aims to eliminate this misconception and facilitate those systemic practices that allow professionals to have a work potential beyond the average.

On the other hand, after qualitatively evaluating some characteristics of "Professional Networking" on the Tegucigalpa context, it is important to assert that according to the empirical evidence, which es supported by several inputs, it is inferred that the main limitation for the effective Management of the "Professional Networking" in the labor market of Tegucigalpa, responds to the low perception of institutional strength, with which the public and private organizations in the country count, a situation that compromises the application of the good practices of "Professional Netwoking" As well as the healthy pursuit of job opportunities.

Finally, this document emphasizes the different characteristics of "Professional Networking" in Honduras, by testing the different principles of the Anthropologist Robin Dunbar along the Tegucigalpa Labor Market reality.

Key words: employment, networking, employment ambience. 


\section{INTRODUCCIÓN}

El complejo reto de insertarse en el mercado laboral hondureño, se acentúa cada vez más, sin limitarse a la edad, género, grado académico o grado de experiencias del profesional; sin embargo para los jóvenes estudiantes, que no han concluido sus estudios y aun no se han enfrentado con esta insípida realidad, es importante, considerar algunas aseveraciones en este análisis, el cual tiende a cuestionar las estadísticas de la Secretaria de Trabajo y Seguridad Social Hondureña, entorno a sus métricas de desempleo, subempleo, tiempos promedio para acceder a un trabajo, rotación de personal, etc. (Alchian, 1950).

Ante las condiciones descritas con anterioridad, es importante inferir, que la intención de este trabajo no es sembrar el pánico entre los futuros profesionales/pasantes del sistema educativo superior hondureño, sin embargo, este análisis

Considerando que era oportuno el estudiar las condicionantes y particularidades del mercado laboral hondureño, para sensibilizarles sobre la importancia de aplicar las buenas prácticas "networking profesional", al caracterizar distintos elementos de este (limitantes, oportunidades y condicionantes), de manera tal, que estos abonen a mitigar el riesgo de no atinar con oportunidades laborales que satisfagan las expectativas de los profesionales, ante la condicionante, de encontrarse ajeno a las redes de contactos profesionales adecuadas, a efecto de potenciar su tejido/matriz de contactos.

Vale pena mencionar que dentro de la evidencia teórica y empírica en la que se amparan la mayoría de estas posturas y hallazgos, figuran:

- ¿Porque fracasan los países? De James Robinson,

- "La Riqueza de las Naciones" de Adam Smith.

- Corrupción y Debilidad Institucional: Factores de Inseguridad de Carolina Sampó.

- "How many Friends does one person need?" de Robin Dunbar.

- Entre otros.

\section{Objetivo general del estudio}

Caracterizar la implementación del networking profesional, en el mercado laboral de Tegucigalpa, Francisco Morazán, en el período 2016-2017. 


\section{Objetivos específicos del estudio}

- Conocer las condicionantes principales del mercado laboral hondureño, para la efectiva implementación del networking profesional.

- Determinar cuáles de las prácticas del "networking profesional", son más certeras en el contexto laboral hondureño.

- Determinar si la base teórica del networking profesional de Robin Dunbar, se aplica en el contexto laboral hondureño.

\section{Fundamentación teórica}

Durante mucho tiempo las empresas y organizaciones en general, han intentado cuantificar el capital intelectual de los individuos, de manera tal que este componente pueda ser registrado y contabilizado, como un activo de tipo intangible, sin embargo, es de enorme importancia el mencionar que pocos son los esfuerzos que los académicos, sociólogos y científicos han desarrollado para medir las potencialidades del capital social, de un individuo en un contexto en particular, el cual según el académico Eduardo Bueno Campos, es hoy en día sinónimo de "nivel de efectividad del networking" (Bueno, 1998).

En este apartado, se abordan las distintas teorías, que sustenta la necesidad del profesional contemporáneo en aplicar de manera regular las distintas prácticas del networking, concentrándonos en la tipología denominada como "networking profesional", el cual es un acelerador y garante del éxito en la carrera profesional de un individuo, como son:

- La teoría del número máximo de contactos efectivos, cuyo principal precursor es el antropólogo Robin Dunbar.

- La teoría de los seis grados de contacto, la cual es impulsada por el periodista Frigyes Karinthy.

- El análisis científico denominado "un pequeño mundo", realizado por el psicólogo y sociólogo norte americano, llamado Stanley Milgram.

De igual manera, estos insumos teóricos, son concatenados con estudios empíricos realizados por Robin Dunbar, Adam Smith y Carolina Sampó, quienes persiguen las causalidades que han estimulado la implementación de las prácticas del networking profesional, en los profesionales contemporáneos.

Al adentrarse en el término definido como "networking", este vocablo etimológica- 
mente indica que es una expresión de origen sajón, nada nueva o novedosa en el argot del mercado laboral, pues según un estudioso del capital social, el historiador Douglas Harper, este concepto empezó a utilizarse desde el año 1947, con el objetivo de representar la interconexión de personas y redes de contactos a estructuras mayores, denominadas matrices de contacto (Dunbar, How many Friends does one person need, 2010). Considerando que el término de networking, no cuenta con una traducción directa al español, esta expresión puede traducirse como: "gestión estratégica de red de contactos", la cual puede también semejar a algunos académicos como el "potenciamiento del capital social". Es importante mencionar que no existe una definición propia del networking, no obstante, dentro de las más aceptadas se encuentra la formulada por Robin Dunbar quien le define como: "Al conjunto de redes que se entrelazan entre sí, según una orientación estratégica de objetivos a corto, mediano o largo plazo, de quien o quienes se lo(s) proponen" (Dunbar, Human Evolutionary Psychology, 2002). Sin embargo, dada la bastedad de su alcance, el concepto de Networking es categorizado por los académicos en 4 clases: networking profesional, networking online, networking presencial y networking corporativo (Qualman, 2009), señalando que este análisis se centraren la caracterización del Networking de tipo Profesional en el contexto local hondureño, el cual define la capacidad de un individuo para insertarse de manera efectiva a una red de contactos de manera efectiva y sostenible.

Luego de introducir el concepto principal de esta pesquisa, se da paso a la descripción de los insumos teóricos recabados por este análisis, referentes a las teorías que originan y dan forma al concepto de "networking", la cual fue modelada por el antropólogo Robin Dunbar, cuya propuesta establece que para cualquier persona, es virtualmente imposible, el construir un conjunto de relaciones estables y fuertemente vinculantes, con un número superior a 150 contactos, fundamentando su criterio en base a limitantes temporales, personales, sociales y económicas del individuo (Dunbar, How many Friends does one person need, 2010).Para Robin Dunbar ese aplicativo es indistinto a cualquier tipo de profesión, área de conocimiento y contexto, generalizando este principio a todo tipo de realidades (tanto las más complejas, como las más homogéneas).

Cabe mencionar que la propuesta de Robin Dunbar, ha influenciado la industria, academia, así como en las redes de profesionales altamente especializados, al grado que, varios académicos han justificado que sus postulados cuentan con poca aplicabilidad, sosteniendo que las redes de contactos efectivos de cualquier profesional, dependen de las realidad puntuales del mercado laboral, ocupación, así como acceso a las redes sociales, basando la mayoría de sus argumentos, en la propuesta de 
James Robinson, quien a través de su publicación denominada: "Porque Fracasan los Países", destaca, que el subdesarrollo de los países y las secuelas de esta condición, no es producto de su ubicación geográfica (como ser el desempleo, entre otros), ni mucho menos de la errada ignorancia de las autoridades políticas de una país, en aplicar y dar seguimiento a las prácticas correctas de gestión administrativa gubernamental, sino en la debilidad sus instituciones (públicas y privadas), que no garantizan el transparente cumplimiento de los principios y/o funciones sobre los cuales fueron creadas. Por lo tanto se alteran las condiciones para que se apliquen de manera transparente, espontánea y plena las prácticas de networking profesional necesarias (Acemoglu \& Robinson, 2013).

Sumado al aporte de Robinson, entorno a la interpretación de la publicación de Adam Smith "La Riqueza de las Naciones" (Acemoglu \& Robinson, 2013), en la cual se explica que el capital social no incide sobre la productividad de un individuo o sobre las potenciales de este en un ámbito de trabajo determinado, en su lugar Smith vincula directamente a dos variables:

- La división del trabajo y adecuada burocratización de los roles y funciones en las organizaciones.

- El grado de intervención gubernamental en el accionar de las instituciones públicas y privadas, para mejorar las condiciones de un libre mercado.

Donde, ambas variables, consideran que el fortalecimiento de la institucionalidad (tanto pública, como privada) es una de las medidas que más urge adoptar, para cambiar las condiciones del subdesarrollo y movimientos demográficos. Destacando que con este postulado se da paso al concepto de demografía empresarial, en la cual se demuestra que existe migración de profesionales y empresas, debido a la saturación de entornos productivos y laborales, producto de escenarios económicos complejos, con pocas posibilidades de aplicar ampliamente los principios de Robin Dunbar, de manera efectiva.Es importante, contrarrestar la óptica de Robinson y Smith, con los aportes del Psicólogo Stanley Milgram, quien desarrolló una secuencia de experimentos a los cuales denominó "Un Pequeño Mundo", En el cual el pretende probar ¿Cómo dos individuos seleccionados al azar podrían llegar a conectarse entre sí, gracias a la adecuada planeación de una estrategia de networking profesional? demostrando que el capital social, siempre puede ser potenciado, independientemente de las limitantes geográficas, del idioma, edad o condición social (Blass, 2002).

Destacando que el análisis de Milgram a inspirado a muchos académicos en la exploración de estas condicionantes y de como el networking tendría que mutar o llegar a 
"tropicalizarse" según la realidad laboral en la cual se definan objetivos a corto, mediano y largo plazo, así como en el área de conocimiento, dentro de las que se destaca el trabajo de Frigyes Karinthy, denominado "La Teoría de los 6 Grados" (Watts, 2006), en la cual se efectuaron varios experimentos con profesionales seleccionados de manera aleatoria, quienes no se conocían entre si y los reunieron por jornadas extensas, para efecto de determinar las rutas en su cadena/red de contactos, en las que ambos individuos coincidieran en un contacto al cual ambos conocían o tenían algún grado de relación, relevando que la mayoría de las personas entrevistadas presentaron una ruta o línea de contactos no mayor a 6 personas, dando paso a la normativa de 6 grados o contactos máximos, para coincidir con una persona en particular; situación que fue desmentida, por el creador de la Red Social FACEBOOK, al demostrar que estas variaciones presentan un sin número de condicionantes sociales y de contexto, reduciéndolo de 6 grados de contacto a 3.57 grados (Ramirez, 2016).

Finalmente, se destaca que una de las principales críticas a los supuestos de Capital Social de Robin Dunbar, se han manifestado a través de la Economista y Socióloga, Carolina Sampó, quien en su obra denominada Corrupción y Debilidad Institucional: Factores de Inseguridad, cuestiona fuertemente que a los modelos sociales propuestos por Dunbar, al reprochar, que el antropólogo supone que todos los contextos sociales son ideales y que las barreras entre las rutas de contactos son universales para todas las realidades (Sampó, 2013). Acentuando en este trabajo, que una de las principales barreras para un networking efectivo, es precisamente la debilidad en las organizaciones/instituciones, al flexibilizar sus procesos y protocolos de gestión, manifestándose en proporciones similares, tanto a nivel público, como en el privado (Qualman, 2009).

Por otro lado, al hablar de las redes, de manera general, es importante el mencionar que estos académicos identificaron a varias tipologías de redes de contactos: como ser:

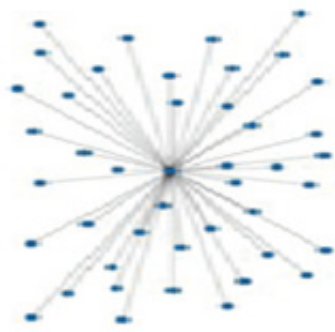

La red centralizada

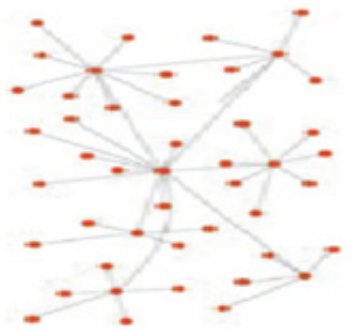

La red descentralizada

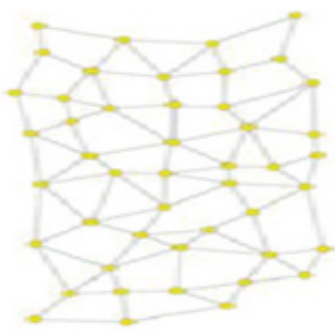

La red distribuida 
Donde las redes Centralizadas, suelen ser aquellas en las que un individuo lidera de manera permanente la actividad social y controla el dinamismo en la red, señalando que este tipo de tejidos de contactos, suelen manifestarse con regularidad en los ámbitos de las empresariales familiares y estructuras empresariales verticalizadas (Dunbar, 2010).

Las redes descentralizadas, son aquellas que se manifiestan en aquellas organizaciones en las cuales que la carga laboral es delegada a distintas unidades de trabajo, quienes cuentan con responsables o líderes de gestión definidos, así como con una vértebra jerárquica más vertical, tal es el caso de las estructuras organizacionales en las campañas políticas (Dunbar, 2010).

Finalmente, las redes homogéneamente distribuidas, en las cuales todos los miembros suelen contar con los mismos canales de comunicación y suelen tener acceso a interactuar con la mayoría de los contactos vigentes en la red misma, al prescindir de canales efectivos de interacción y un sentido de pertenencia a la red de contactos a la cual se pertenece (Davenport, 1996).

\section{METODOLOGÍA}

Se destaca, que la naturaleza de esta pesquisa es de este tipo cualitativo, en la cual se procuró estudiar el fenómeno del networking profesional, sobre 88 profesionales, de manera transversal, con el apoyo de un grupo de expertos en la temática, con características específicas, cuyas tipologías contaban con 4 perfiles:

- Un grupo de expertos perteneciente a un sector del mercado laboral, en el cual las condiciones son permanentes, desempeñándose en ámbito gerencial y/o de gestión de recursos humanos, con un margen temporal no menor a 3 años en el cargo. A fin de determinar si la teoría de Robin Dunbar es válida, independientemente del cargo que ostenta el profesional en la organización.

- Otro grupo de expertos en la categoría de especialistas libres, a nivel de consultorías, gerentes de proyectos a nivel senior, técnicos freelance, médicos especialistas, etc., con el objetivo de verificar, si pese a no formar parte de una estructura permanente de trabajo, el networking profesional siempre es aplicable.

- El tercer grupo de profesionales entrevistado, es un grupo de empresarios, quienes son los encargados de monitorear las rotaciones, contrataciones y rendimiento de su personal, al igual que los profesionales que laboran en empleos 
permanentes, el análisis de esta categoría tuvo la intención de probar si el networking profesional, se intensifica según la posición desempeñada en la organización (independientemente de cuan vertical u horizontal sea la burocratización de sus roles).

- El cuarto y última categoría de expertos analizada en esta pesquisa, la cual contempla a un grupo de académicos y autoridades universitarias, quienes facilitan una óptica neutral del networking, desde la óptica de la oferta y formación de profesionales en el país. Formado, con el objetivo de valorar la importancia del networking profesional, en los profesionales recién egresados.

Cabe mencionar, que de los 88 profesionales con trayectorias profesionales reconocidas, inicialmente mapeados, se levantaron insumos de 52 de ellos(as) en las siguientes proporciones: 20 gestores del recurso humano, 16 médicos especialistas, 9 empresarios, 7 autoridades universitarias (pertenecientes al sector privado).

Señalando, que los gestores de talento humano y autoridades universitarias, pertenecen a los más altos niveles de gestión, en las escalas jerárquicas de sus respectivas instituciones, con trayectorias profesionales que superan los 10 años de experiencia en puestos de alta gerencia.

La unidad de análisis, en el estudio, refiere al profesional universitario que ejerce en el mercado laboral de Tegucigalpa en la actualidad, ocupando un rol a nivel gerencial. Respecto al instrumento de medición, cabe mencionar que la construcción del mismo se realizó en cuatro etapas, entre las cuales figuran:

1) El planteamiento de las preguntas en acorde a los objetivos, alcance y dimensiones de la variable en análisis (networking).

2) Se procedió a filtrar las interrogantes, según su estructura y planteamiento.

3) Se desarrolló un proceso de validación de contenido de las preguntas, según 4 criterios:

- Relevancia de las preguntas.

- Claridad de las preguntas.

- Coherencia entre las preguntas.

- Suficiencia de las preguntas formuladas (es decir, si los temas en análisis por cada pregunta son suficientes, para responder al alcance de los objetivos previamente definidos).

4) Se filtró la herramienta, según los insumos recabados de la validación de contenido y se definieron los perfiles de los informantes, determinando 4 categorías de entrevistados. 
Se describen a continuación los perfiles de los entrevistados, según la estrategia metodológica abordada (ver Tabla $\mathrm{N}^{\circ} 1$ ).

\section{Tabla 1. Perfiles de los informantes seleccionados}

\begin{tabular}{|c|c|c|c|}
\hline & Condiciones del perfil & Objetivo de análisis & $\begin{array}{l}\text { Cantidad mínima de } \\
\text { entrevistados }\end{array}$ \\
\hline $\begin{array}{l}\text { Grupo de } \\
\text { académicos }\end{array}$ & $\begin{array}{l}\text { Docentes y autoridades } \\
\text { universitarios, que cuentan } \\
\text { con amplio conocimiento del } \\
\text { mercado laboral hondureño. }\end{array}$ & $\begin{array}{l}\text { Valorar la importancia del } \\
\text { networking profesional, en los } \\
\text { profesionales recién egresados } \\
\text { desde la academia. }\end{array}$ & $\begin{array}{l}\text { Hasta que se alcance } \\
\text { la saturación teórica } \\
\text { de esta categoría de } \\
\text { informante, la cual se } \\
\text { alcanzó al cabo de los } \\
7 \text { entrevistados. }\end{array}$ \\
\hline $\begin{array}{l}\text { Grupc } \\
\text { profesio } \\
\text { indepeno }\end{array}$ & $\begin{array}{l}\text { Profesionales con plazas de } \\
\text { trabajo temporales, que } \\
\text { laboran como agentes libres, } \\
\text { a nivel de consultorías, } \\
\text { gerentes de proyectos a nivel } \\
\text { senior, técnicos freelance, } \\
\text { médicos especialistas, etc. }\end{array}$ & $\begin{array}{l}\text { Verificar, si pese a no formar parte } \\
\text { de una estructura permanente de } \\
\text { trabajo, el networking profesional } \\
\text { siempre es aplicable. }\end{array}$ & $\begin{array}{l}\text { Hasta que se alcance } \\
\text { la saturación teórica } \\
\text { de esta categoría de } \\
\text { informante, la cual se } \\
\text { alcanzó al cabo de los } \\
16 \text { entrevistados. }\end{array}$ \\
\hline $\begin{array}{l}\text { Grupo de } \\
\text { profesionales a } \\
\text { nivel Gerencial } \\
\text { en Empleos } \\
\text { Fijos }\end{array}$ & $\begin{array}{l}\text { Profesionales } \\
\text { desempeñándose en ámbito } \\
\text { gerencial y/o de gestión de } \\
\text { recursos humanos, con un } \\
\text { margen temporal no menor a } \\
3 \text { años en el cargo, con plazas } \\
\text { laborales permanentes. }\end{array}$ & $\begin{array}{l}\text { Determinar si la teoría de Robin } \\
\text { Dunbar es válida, } \\
\text { independientemente del cargo que } \\
\text { ostenta el profesional en la } \\
\text { organización. }\end{array}$ & $\begin{array}{l}\text { Hasta que se alcance } \\
\text { la saturación teórica } \\
\text { de esta categoría de } \\
\text { informante, la cual se } \\
\text { alcanzó al cabo de los } \\
20 \text { entrevistados. }\end{array}$ \\
\hline $\begin{array}{l}\text { Grupo de } \\
\text { empresarios }\end{array}$ & $\begin{array}{l}\text { Empresarios hondureños, } \\
\text { cuyas empresas radican en el } \\
\text { Departamento de Francisco } \\
\text { Morazán, Honduras. }\end{array}$ & $\begin{array}{l}\text { Probar si el networking profesional, } \\
\text { se intensifica según la posición } \\
\text { desempeñada en la organización } \\
\text { (independientemente de cuan } \\
\text { vertical u horizontal sea la } \\
\text { burocratización de sus roles). }\end{array}$ & $\begin{array}{l}\text { Hasta que se alcance } \\
\text { la saturación teórica } \\
\text { de esta categoría de } \\
\text { informante, la cual se } \\
\text { alcanzó al cabo de los } \\
9 \text { entrevistados. }\end{array}$ \\
\hline
\end{tabular}

Fuente: Información levantada en el análisis, expertos Hondureños en el área de networking

En la tabla 1, se manifiesta la estrategia con la cual se seleccionó a las 4 tipologías de profesionales, descartando a otro tipo de categorías de expertos, al considerar que la principal razón de su selección, se fundamentó en el objetivo específico, que busca probar la universalidad de la teoría de Robin Dunbar en el contexto laboral hondureño.

Finalmente, más allá de la estrategia cualitativa que se utilizó, es relevante para este apartado metodológico el resaltar, que durante la recopilación de los insumos teóricos y empíricos, se procura efectuar un contraste entre las distintas realidades a nivel mundial y como el fenómeno del networking profesional, se implementa, según estas características muy particulares de estos contextos. De igual manera, es importante mencionar que existen 4 tipos/dimensiones del networking: 
- Networking profesional.

- Networking virtual.

- Networking presencial.

- El Networking corporativo.

Sin embargo para efectos de este análisis, solamente se estará analizando el Networking de Tipo Profesional.

\section{RESULTADOS}

Dentro de los resultados encontrados en este análisis se destaca lo siguiente:

Objetivo específico $n^{\circ} 1$ : Al considerar que la tasa de desempleo en el país, oscila en alrededor de $7.31 \%$, con la particularidad de que la tasa de desempleo abierto (TDA) para las personas que cuentan con un nivel educativo/superior, es de alrededor de $9.93 \%$, y aquellos ciudadanos, con educación básica presentan un TDA de $5.28 \%$, es válido inferir, que los individuos con un nivel académico superior, cuentan con potencialmente menos posibilidades de adquirir un trabajo. Destacando que el uso de un efectivo networking profesional, se vuelve casi obligatorio para el profesional contemporáneo. Es decir que contradictoriamente, mientras más especializado se encuentra un profesional en el mercado laboral de Tegucigalpa, menos posibilidades tendrá éste de insertarse, debido a la poca capacidad de inserción que presentan las empresas para remunerarle.

Objetivo específico $\mathrm{No}^{\circ}$ 2: Es importante el destacar que dentro de las prácticas más certeras y recomendadas, por los expertos entrevistados en la pesquisa, resalta que para hacer el networking profesional más efectivo se necesita:

- La constante autoevaluación, autocritica y verificación del cumplimiento de las metas a corto y mediano plazo, para confirmar, si se ha incursionado en la red de contactos adecuada.

- La regular capacitación sobre competencias específicas, que le ayuden al profesional a destacarse del resto de sus colegas.

- La participación regular del profesional en voluntariados, pasantías/internships y demás actividades extracurriculares, que abonen a su experiencia del mismo, así como al fortalecimiento de su hoja de vida.

- En el contexto laboral hondureño la visibilidad en las redes sociales, presentan un 
potencial de gestión laboral bien limitado, el cual es menos provechoso, que el establecimiento de las relaciones interpersonales, la cual estimula la creación de contactos específicos, generando un ambiente de confianza y pertinencia a la red profesional.

- El fortalecimiento de las habilidades interpersonales, que ayudan a:

* Leer coyunturas y adelantarse a posibles oportunidades laborales.

* Fortalecer los nexos entre los contactos uno a uno y colectivamente.

* Acelerar los vínculos de confianza y pertinencia a la red de contactos.

Objetivo específico $\mathrm{N}^{\circ}$ 3: Dentro de los hallazgos más relevantes en este análisis, se infiere que en el marco del mercado laboral de Tegucigalpa, la teoría del "número de Dunbar", enunciado por el antropólogo Robin Dunbar, no aplica, al variar en resultado, dependiendo del rol y rubro en el cual un individuo se desarrolla, al poder exponer el supuesto de a expertos de distintas áreas de conocimiento y rubros de gestión (revisar Tabla $\mathrm{N}^{\circ}$ 2). Dando como resultado lo siguiente:

Tabla 2. Rangos de contactos promedio según la categorización de los profesionales en el mercado laboral Hondureño.

\begin{tabular}{|c|c|l|}
\cline { 2 - 3 } \multicolumn{1}{c|}{} & $\begin{array}{c}\text { Rango de contactos promedio } \\
\text { en distintas redes sociales }\end{array}$ & $\begin{array}{c}\text { Justificación de los expertos } \\
\text { entrevistados, sobre número de } \\
\text { Contactos }\end{array}$ \\
\hline $\begin{array}{c}\text { Profesionales } \\
\text { con cargos } \\
\text { Gerenciales }\end{array}$ & $120-140$ & $\begin{array}{l}\text { Cuentan con la media de relaciones } \\
\text { interpersonales, propuesta por Robin } \\
\text { Dunbar. }\end{array}$ \\
\hline $\begin{array}{c}\text { Profesionales } \\
\text { Freelance/Cons } \\
\text { ultores/Indepen } \\
\text { dientes }\end{array}$ & $140-180$ & $\begin{array}{l}\text { Estos profesionales, requieren de una } \\
\text { cantidad superior de contactos, que } \\
\text { aquellos que tienen empleo fijo, para } \\
\text { mitigar el riesgo de gestión por contrato. }\end{array}$ \\
\hline $\begin{array}{c}\text { Empresarios } \\
\text { Académicos }\end{array}$ & $150-200$ & $\begin{array}{l}\text { Los Empresarios requieren de una } \\
\text { cantidad superior de contactos, porque su } \\
\text { gestión se fundamenta en la cadena de } \\
\text { valor y suministro a la cual pertenece. }\end{array}$ \\
\hline \multirow{2}{*}{$\begin{array}{c}\text { Eos académicos requieren de una } \\
\text { cantidad importante de contactos ,para el } \\
\text { desarrollo de investigación colaborativa, } \\
\text { así como en la articulación docencia y la } \\
\text { práctica, sin embargo esta se encuentra } \\
\text { dentro de la media estándar de relaciones } \\
\text { planteada por Robin Dunbar. }\end{array}$} \\
\hline
\end{tabular}

Fuente: Interpretación de los Insumos recabados de las entrevistas aplicadas. 
Un elemento a resaltar en el proceso cualitativo de caracterización del fenómeno del networking en el mercado laboral hondureño, es el hecho de que la teoría del antropólogo Robin Dunbar, no resultó aplicar en dicho entorno, señalando que esta propuesta plantea que para todo individuo existen condicionantes de tiempo, así como de recursos, para alinear una efectiva relación con más de 150 contactos, en un marco temporal definido. En este análisis se levantó información de 4 tipologías de profesionales, que potencialmente podrían no acompañar el precepto de Dunbar y así fue, tal es el caso de los profesionales con cargos gerenciales: los cuales engloban a individuos exitosos, asalariados, con empleos fijos/ a tiempo completo, que se han desempeñado con éxito en ramas específicas de las ciencias administrativas, los cuales persiguen un crecimiento sostenible en la organización, así como un rendimiento constante en los roles y funciones asignadas, sin embargo, para conseguir lo enunciado con anterioridad deben de delegar responsabilidades a distintas personas y para esto tendrán que estar en constante contacto con sus subordinados, construyendo una red de contactos, basada en la confianza, ética y profesionalismo.

En el caso de los profesionales categorizados como freelance / consultores / especialistas independientes, es importante mencionar que en esta tipología, se engloban profesionales como médicos especialistas, consultores senior de proyectos varios, profesionales en la informática y demás áreas del conocimiento que pertenecen a grupos de consultoría legalmente constituidos, etc. Señalando que son precisamente estos profesionales quienes manifiestan, que para su sostenibilidad financiera y laboral, es importante el mantener un red de contacto a nivel local e internacional importante, situación que deja mal parado al supuesto de Robin Dubar, superando la cantidad de contactos efectivos necesarios para postularse a opciones laborales atractivas.

Ahora bien, estos mismos profesionales no niegan que en algún momento en sus carreras, era para ellas(os) complicado el superar un volumen de 150 contactos efectivos de manera sostenible, sin embargo, la construcción de relaciones fuertes, así como el desarrollo de una marca personal destacada, abonó a su bienestar actual. Al evaluar a la categoría de expertos en el tema del networking, denominada como empresarios, cabe mencionar que el perfil entrevistado, fueron mujeres y hombres de negocios, con una trayectoria superior a 5 años de gestión y un grado/certificación académica de Educación Superior, que respalde sus decisiones y conocimiento, quienes denotaron, que hoy en día, para cualquier empresario o joven emprendedor, es "imposible" sostener una organización privada sin el apoyo de una efectiva red de networking que respalde la cadena de valor y suministro de su negocio, permitiéndole dedicarse plenamente a sus actividades en las cuales la institución ha centrado sus esfuerzos para abordar a su mercado meta. Por otro lado, el networking, con sus 
empleados es vital, porque genera confianza y fortalece el sentido de pertenencia de todos con la organización.

Para finalizar, se destaca la óptica de los académicos entorno a la temática del networking profesional, señalando que estas autoridades universitarias, pese a validar el precepto de Robin Dunbar (al contar con un número promedio menor a los 150 contactos en sus redes), mencionan, que el networking, es hoy en día una poderosa herramienta para las universidades a nivel investigativo, la cual tiene un potencial de gran alcance. Otro elemento que se destaca en los resultados fueron las distintas opiniones vertidas sobre el fenómeno del networking profesional, a nivel de las 4 tipologías, las cuales están resumidas en las Tablas $\mathrm{N}^{\circ} 3$ y $\mathrm{N}^{\circ} 4$ a continuación:

Tabla 3. Principales insumos de las entrevistas semiestructuradas, preguntas 1-5

\begin{tabular}{|c|c|c|c|c|c|}
\hline & Pregunta $N^{\circ} 1$ & Pregunta $N^{\circ} 2$ & Pregunta $N^{\circ} 3$ & Pregunta $N^{\circ} 4$ & Pregunta $\mathbf{N}^{\circ} 5$ \\
\hline $\begin{array}{l}\text { Grupo de } \\
\text { académicos }\end{array}$ & $\begin{array}{l}\text { El mercado laboral } \\
\text { hondureño esta } \\
\text { convulsionado, } \\
\text { acondicionado por la } \\
\text { falta de inversión } \\
\text { local y extranjera en } \\
\text { el mismo. }\end{array}$ & $\begin{array}{l}\text { Es una estrategia de } \\
\text { visibilización } \\
\text { profesional que } \\
\text { potencia la rápida } \\
\text { inserción de los } \\
\text { profesionales. }\end{array}$ & $\begin{array}{l}\text { El Networking se } \\
\text { presenta en } \\
\text { ambos contextos, } \\
\text { sin embargo, no } \\
\text { se manifiesta de } \\
\text { manera directa en } \\
\text { el ámbito público, } \\
\text { sino que a nivel } \\
\text { de otras } \\
\text { tipologías. } \\
\end{array}$ & $\begin{array}{l}\text { El desarrollo de } \\
\text { actividades extra } \\
\text { curriculares de apoyo } \\
\text { académico, social e } \\
\text { investigativo que nutra } \\
\text { la hoja de vida del } \\
\text { futuro profesional, } \\
\text { siempre estar en } \\
\text { constante formación. }\end{array}$ & $\begin{array}{l}\text { Tener los objetivos } \\
\text { bien claros, junto } \\
\text { con el } \\
\text { fortalecimiento a } \\
\text { valores. }\end{array}$ \\
\hline $\begin{array}{l}\text { Grupo de } \\
\text { profesionales } \\
\text { independientes }\end{array}$ & $\begin{array}{l}\text { A dejado de ser un } \\
\text { mercado laboral } \\
\text { como tal, en su lugar } \\
\text { se convertido en una } \\
\text { pequeña laguna de } \\
\text { oportunidades, en } \\
\text { donde aquel que } \\
\text { este mejor adherido } \\
\text { a una red, sea } \\
\text { política o profesional } \\
\text { será el favorecido. }\end{array}$ & $\begin{array}{l}\text { Es una matriz } \\
\text { profesional de } \\
\text { expertos, que } \\
\text { permite a sus } \\
\text { miembros } \\
\text { intercambiar datos } \\
\text { experiencias, } \\
\text { conocimiento y } \\
\text { oportunidades de } \\
\text { negocio y trabajo. }\end{array}$ & $\begin{array}{l}\text { En Honduras } \\
\text { ambos entornos } \\
\text { están viciados. }\end{array}$ & \begin{tabular}{|l} 
El \\
ventilarse/visibilizarse \\
más en las redes \\
sociales e identificarse \\
más con los grupos \\
afines a los objetivos \\
trazados.
\end{tabular} & $\begin{array}{l}\text { Tener los objetivos } \\
\text { bien definidos y ser } \\
\text { paciente, así como } \\
\text { de manejar los } \\
\text { tiempos de } \\
\text { visibilización y } \\
\text { gestión. }\end{array}$ \\
\hline $\begin{array}{l}\text { Grupo de } \\
\text { profesionales a } \\
\text { nivel gerencial } \\
\text { en empleos } \\
\text { fijos }\end{array}$ & $\begin{array}{l}\text { El mercado laboral } \\
\text { hondureño, cuenta } \\
\text { con una débil } \\
\text { institucionalidad, la } \\
\text { cual se manifiesta } \\
\text { de la misma manera, } \\
\text { tanto el ámbito } \\
\text { público, como en el } \\
\text { privado. }\end{array}$ & $\begin{array}{l}\text { Es una red de } \\
\text { contactos, que } \\
\text { facilita al profesional } \\
\text { adquirir nuevas } \\
\text { experiencias y } \\
\text { competencias en } \\
\text { áreas de } \\
\text { conocimiento } \\
\text { determinadas. }\end{array}$ & $\begin{array}{l}\text { En Honduras } \\
\text { ambos entornos } \\
\text { están viciados. }\end{array}$ & $\begin{array}{l}\text { El contar con más } \\
\text { visibilidad, tanto en las } \\
\text { plataformas de trabajo } \\
\text { digitales, como en las } \\
\text { redes de profesionales } \\
\text { de su área. }\end{array}$ & $\begin{array}{l}\text { Tener los Objetivos } \\
\text { Bien definidos y } \\
\text { trazados. }\end{array}$ \\
\hline $\begin{array}{l}\text { Grupo de } \\
\text { Empresarios }\end{array}$ & $\begin{array}{l}\text { El mercado laboral } \\
\text { hondureño se } \\
\text { encuentra dañado } \\
\text { por el riesgo de } \\
\text { inversión y alta } \\
\text { inseguridad. }\end{array}$ & $\begin{array}{l}\text { Es la técnica } \\
\text { empleada por un } \\
\text { profesional o } \\
\text { institución para } \\
\text { romper con las } \\
\text { barreras del sistema } \\
\text { empresarial/mercado } \\
\text { laboral. }\end{array}$ & $\begin{array}{l}\text { El sector público } \\
\text { es en el que más } \\
\text { se acentúan } \\
\text { estos fenómenos. }\end{array}$ & $\begin{array}{l}\text { La capacitación } \\
\text { constante y la } \\
\text { visibilización de sus } \\
\text { logros, tanto } \\
\text { profesionales como } \\
\text { académicos son } \\
\text { vitales. }\end{array}$ & $\begin{array}{l}\text { Es una mezcla entre } \\
\text { tener Los Objetivos } \\
\text { Claros y saberse } \\
\text { vender (tener } \\
\text { buenas actitud } \\
\text { buenas } \\
\text { presentación). }\end{array}$ \\
\hline
\end{tabular}

Fuente: Interpretación de las entrevistas aplicadas a distintos profesionales, pertenecientes a 4 categorías de gestión laboral. 
Tabla 4. Principales insumos de las entrevistas semiestructuradas, preguntas 6-9

\begin{tabular}{|c|c|c|c|c|}
\hline & Pregunta $\mathrm{N}^{\circ} 6$ & Pregunta $\mathrm{N}^{\circ} 7$ & Pregunta $N^{\circ} 8$ & Pregunta $\mathrm{N}^{\circ} 9$ \\
\hline $\begin{array}{l}\text { Grupo de } \\
\text { académicos }\end{array}$ & $\begin{array}{l}\text { La marca personal, habla de } \\
\text { constancia en todos los ámbitos, } \\
\text { regularidad y congruencia entre } \\
\text { la imagen que se proyecta con } \\
\text { los actos que se realiza. }\end{array}$ & $\begin{array}{l}\text { El desarrollo de actividades } \\
\text { extracurriculares, acompañada } \\
\text { por certificados académicas } \\
\text { que respalden que se cuenta } \\
\text { con un conocimiento en el } \\
\text { área. }\end{array}$ & $\begin{array}{c}\text { Entre } 120-150 \\
\text { contactos }\end{array}$ & $\begin{array}{l}\text { El networking, junto } \\
\text { con la marca } \\
\text { personal que me } \\
\text { antecede ha sido } \\
\text { vital en mi carrera } \\
\text { profesional. } \\
\end{array}$ \\
\hline $\begin{array}{c}\text { Grupo de } \\
\text { profesionales } \\
\text { independientes }\end{array}$ & $\begin{array}{l}\text { La marca personal de la persona } \\
\text { está sujeta a cuanto se pueden } \\
\text { acentuar los hitos en su carrera } \\
\text { profesional, en la medida en que } \\
\text { estos se pueden nombrar en } \\
\text { línea de tiempo. }\end{array}$ & $\begin{array}{l}\text { El cúmulo de experiencias, en } \\
\text { áreas relevantes, es } \\
\text { importante, si desarrollan } \\
\text { estas ad honorem, es más } \\
\text { reconocido por las } \\
\text { empresas/organizaciones. }\end{array}$ & $\begin{array}{l}\text { Entre } 140-180 \\
\text { contactos }\end{array}$ & $\begin{array}{l}\text { Por la naturaleza de } \\
\text { mi carrera, es } \\
\text { importante el } \\
\text { desarrollo de un } \\
\text { buen networking y } \\
\text { vinculación efectiva } \\
\text { con mis pacientes. } \\
\end{array}$ \\
\hline $\begin{array}{l}\text { Grupo de } \\
\text { profesionales a } \\
\text { nivel gerencial } \\
\text { en empleos } \\
\text { fijos }\end{array}$ & $\begin{array}{l}\text { El autoconocimiento es vital para } \\
\text { que un individuo se supere, } \\
\text { debido a que más allá de la } \\
\text { imagen que proyecta es esencial } \\
\text { el saber en qué mejorar. }\end{array}$ & $\begin{array}{l}\text { El saber escuchar e interpretar } \\
\text { los signos potenciales } \\
\text { oportunidades y de formar } \\
\text { estrategias, congruentes con } \\
\text { esos objetivos. }\end{array}$ & $\begin{array}{c}\text { Entre } 120-140 \\
\text { Contactos }\end{array}$ & $\begin{array}{l}\text { El networking, junto } \\
\text { con la marca } \\
\text { personal que me } \\
\text { antecede ha sido } \\
\text { vital en mi carrera } \\
\text { profesional } \\
\end{array}$ \\
\hline $\begin{array}{c}\text { Grupo de } \\
\text { empresarios }\end{array}$ & $\begin{array}{l}\text { La marca personal es el similar a } \\
\text { una línea de tiempo individual, } \\
\text { en cada profesional, en la cual } \\
\text { se visualiza cuan definidos están } \\
\text { los objetivos del individuo. }\end{array}$ & $\begin{array}{l}\text { La visibilidad, es importante, } \\
\text { para un posicionamiento } \\
\text { adecuado, mientras más } \\
\text { presencia tenga un individuo } \\
\text { en los medios es mejor para él } \\
\text { o ella. }\end{array}$ & $\begin{array}{c}\text { Entre } 150-200 \\
\text { contactos }\end{array}$ & $\begin{array}{l}\text { En el ámbito } \\
\text { empresarial, el } \\
\text { networking es } \\
\text { imprescindible. }\end{array}$ \\
\hline
\end{tabular}

Fuente: Interpretación Personal. Datos recabados de distintas entrevistas a 4 tipologías de profesionales, presente análisis.

\section{CONCLUSIONES}

Luego de explorar el sustento teórico y empírico, sumado a la caracterización cualitativa del fenómeno denominado como networking profesional, en el mercado laboral hondureño, es imperativo el inferir de manera definitiva sobre los hallazgos, levantados por esta pesquisa, trazados por el alcance de la misma, los cuales se reflejan a continuación:

Los factores que más potencian el networking profesional de un individuo en el mercado laboral de Tegucigalpa son:

- La correcta definición y seguimiento de los objetivos a corto y mediano plazo, contemplados por la persona, el cual deberá de estar sujeto a una planificación temporal bien definida.

- El segundo punto que resaltan los expertos del networking hondureño, es el de manejar los tiempos de gestión efectiva del networking y de ser paciente, al entender a plenitud una coyuntura, que permita una mejor lectura de las potenciales oportunidades laborales/ emprendimiento. 
Por otro lado, es importante mencionar que dentro de las principales condicionantes existentes en el mercado laboral de Tegucigalpa, para un efectivo networking profesional se destacan dos apartados:

- Una frágil institucionalidad, la cual se acentúa de manera equitativa tanto en el sector privado, como en el sector público, obstaculizando la meritocracia, deteriorando la competitividad organizacional e impidiendo que los protocolos del networking profesional, se desarrollen a plenitud.

- De igual manera la segunda condicionante que se destaca en el mercado laboral local, responde a que estos profesionales en mención, no están desarrollando vínculos personales con los contactos de interés, dejando todo su esfuerzo y esperanzas de networking, a las redes sociales y bases de datos laborales, que en su lugar, despersonalizan un sistema, que es altamente único y que debe de estimular la confianza entre ambas partes (la red de contactos por sí mismas y el individuo con la red de contactos).

Por otro lado, al explorar la evidencia teórica y empírica sobre la temática del networking profesional, se señalan varias prácticas para el desarrollo de un networking efectivo, sin embargo, son pocas las prácticas que realmente aplican y funcionan en ámbito, del mercado laboral hondureño y de manera particular en Tegucigalpa, como son:

- La adhesión a prácticas, pasantías ad honorem en organizaciones afines a las áreas de conocimiento o interés del profesional.

- La participación del profesional en actividades extracurriculares de bienestar social.

- La formación o capacitación continua, de los profesionales, con la finalidad de fortalecer competencias específicas.

- La obtención de reconocimientos laborales, donde se destaquen resultados 0 metas alcanzadas.

Es válido concluir, que luego de poner a prueba varios puntos enunciados por la teoría de vinculación profesional (antropólogo Robin Dunbar), en el mercado laboral hondureño, se señala que la universalidad de esta propuesta no aplica en ámbito local, debido distintas particularidades. Por lo tanto, el "número de Dunbar" como se le conoce, coincide con la realidad hondureña. Las cuales demostraron superar los 150 contactos, independientemente del nivel jerárquico al cual pertenecen 0 del grado de verticalidad/horizontalidad de la organización (en el mercado laboral hondureño). 


\section{REFERENCIAS BIBLIOGRAFÍCAS}

Acemoglu, D., \& Robinson, J. (2013). Porque fracasan los Paises. Barcelona: Ediciones Deusto.

Alchian, A. (1950). Uncertainty, evolution, and economic theory. Journal of Political Economy, 58: $211-221$.

Blass, T. (2002). The Man Who Shocked the World. Psychology Today, 35(2).

Bueno, E. (1998). El Capital Intangible como Clave Estrategica en la Competencia Actual. Madrid, España: Boletin de Estudio Economicos (No 164, LII) Instituto Complutense de Madrid.

Davenport, T. (1996). Improving Knowledge work Processes. Sloan Management Review, 53-66.

Dunbar, R. (2002). Human Evolutionary Psychology. New Jersey, US: Macmillan and Princeton University Press.

Dunbar, R. (2010). How many Friends does one person need. Liverpool, UK: Faber and Faber.

Qualman, E. (2009). How Social Media Transforms the Way We Live and Do Business. Michigan, US: John Wiley and Sons.

Ramirez, A. (15 de Mayo de 2016). Expertos en marca. Obtenido de www.expertosenmarca.com/facebook-a

\section{ANEXOS}

\section{Anexo $N^{\circ} 1$ Instrumento Cualitativo de Investigación}

Instrumento de Investigación:

1) A su parecer ¿Cuáles son las principales deficiencias/condicionantes del mercado laboral en Honduras?

2) ¿Cómo definiría usted el término de networking?

3) ¿Siente usted que el fenómeno del networking se acentúa más en el ámbito organizacional publico o en el contexto organizacional privado?, ¿Por qué?

4) Según su área de expertise, ¿Cúales son las 5 principales prácticas que todo profesional en el país debe de emplear para practicar un buen "networking"? y ¿Por qué?

5) Siendo estos los indicadores clave de rendimiento o "Key Performance Indicators" (KPI's) del networking:

- Buena actitud

- Tener objetivos bien definidos 
- Presentación

- Saber escuchar

- Ser paciente y manejar los tiempos

- Saber Interactuar

¿A cuál de los anteriores le daría un mayor peso o valor para la obtención de resultados positivos, describa porque?

6) ¿Qué recomendaciones le daría usted a un profesional para mejorar su marca personal?

7) ¿Qué estrategias recomendaría a los nuevos profesionales, para efecto de practicar un buen networking en el ambiente laboral?

8) ¿Podrían ser las redes sociales el mejor medio de comunicación para fortalecer el networking de un profesional? Favor describa por qué sí o porque no.

a. Cuál es la media de contactos comparte en las redes sociales, como Facebook, twitter, etc. ¿Por qué tantos o por qué tan pocos?

b. En un espacio de un mes, ¿Con cuántos de sus contactos en las redes sociales (sean twitter, Facebook), realmente interactúa? (Dunbars number=150)

9) ¿Ha incidido el networking en su éxito laboral/empresarial? Favor describir porque sí/porque no. 